\title{
Unitarity as preservation of entropy and entanglement in quantum systems
}

\author{
Florian Hulpke, ${ }^{1}$ Uffe V. Poulsen, ${ }^{2}$ Anna Sanpera, ${ }^{1,3}$, 田 Aditi Sen(De),${ }^{1,4}$ Ujjwal Sen,,${ }^{1,4}$ and Maciej Lewenstein ${ }^{1,4, \text {, }}$ \\ ${ }^{1}$ Institut für Theoretische Physik, Universität Hannover, D-30167 Hannover, Germany \\ ${ }^{2}$ Department of Physics and Astronomy, University of Aarhus, DK-8000 Aarhus C., Denmark \\ ${ }^{3}$ Grup de Física Teòrica, Universitat Autònoma de Barcelona, E-08193 Bellaterra, Spain \\ ${ }^{4}$ ICFO-Institut de Ciències Fotòniques, Jordi Girona 29, E-08034 Barcelona, Spain
}

\begin{abstract}
The logical structure of Quantum Mechanics (QM) and its relation to other fundamental principles of Nature has been for decades a subject of intensive research. In particular, the question whether the dynamical axiom of QM can be derived from other principles has been often considered. In this contribution, we show that unitary evolutions arise as a consequences of demanding preservation of entropy in the evolution of a single pure quantum system, and preservation of entanglement in the evolution of composite quantum systems. ${ }^{1}$
\end{abstract}

The standard axiomatic formulation of Quantum Mechanics (QM) relies on a set of postulates describing the state of a physical system, its time evolution, and the information that one can gather about the system by performing measurements. A specific formulation for joint quantum systems - beyond the appropriate extension of the postulates - is not regarded in such axiomatic approximation. However, one of the most genuine and counterintuitive properties of quantum mechanics - entanglement - appears only in joint quantum systems. This quantum "inseparability" had for many decades questioned the logical structure of QM and raised a fundamental philosophical debate [1]. The most famous apparent "paradox" aroused by the nonlocal character of composite quantum systems, is exposed in the celebrated article of Einstein, Podolsky and Rosen [2] (the so-called EPR paradox), in which the authors claimed that in any plausible physical theory there exist "elements of physical reality" and questioned the completeness of QM by analyzing entangled states. Afterwards Bell proved in his seminal contributions [3] that if one assumes the validity of "Einstein locality", and an underlying hidden variable theory, there is an upper limit to the correlation between distant events. The claim, in itself, had nothing to do with QM, being a general statement about all physical theories that have an underlying hidden variable model and respects Einstein locality. However, QM predicts the existence of certain entangled states whose correlations (as predicted by QM) violate the upper limit set by Bell (Bell inequalities). Violation of the Bell inequalities showed that the conjunction of the principle of locality (Einstein locality) and the principle of realism (existence of a hidden variable model) is incompatible with QM. After the discovery of the Bell inequalities, experiments were carefully performed to check the predictions of QM regarding the correlations in entangled states. However, the results of all the experiments performed till date to

\footnotetext{
*Also at: Institució Catalana de Recerca i Estudis Avançats.

1 We would also like to dedicate this work to the memory of Asher Peres, whose contributions and sharp comments guided the first steps of the present article.
}

check for violation of Bell inequalities can be described by local hidden variable models, by taking advantage of the inefficiencies of experimental apparatuses. These effects have come to be known as "loopholes". E. Santos was one of the first to point this out [4] (see also [5], and references therein). Curiously enough, in the last decade, this puzzling character of entangled states has become the seed of an emerging technological revolution, whose consequences are hard to overestimate.

The aim of the present contribution is to establish a link between the axioms of QM and the consequences of demanding (i) preservation of entropy in the evolution of a single pure quantum system and (ii) preservation of entanglement in the evolution of composite quantum systems. We shall demonstrate that any of these requirements (either (i) or (ii)), together with other premises, retrieves the dynamical postulate of QM, stating that the evolution of a quantum system follows the Schrödinger equation, i.e. the time evolution must be unitary [6]. In both cases, the converse is obviously true. The paper is organized as follows. In Sec. \ we briefly introduce the axiomatic formalism of QM and our starting premises to derive the dynamical postulate of QM from entropic assumptions and probabilistic linearity. We study maps (transformations) that preserve disorder (entropy) and are linear on mixtures of density matrices. Sec. II deals with preservation of entanglement in composite (bipartite) pure quantum systems. There, we investigate properties of linear maps which preserve entanglement either qualitatively or quantitatively. We demonstrate that every linear map that preserves entanglement, has to be local, or local after a swap operation. We also show that if the preservation of entanglement is quantitative, then the map is a local unitary, or a multiple of a local unitary, after a swap operation.

\section{SINGLE QUANTUM SYSTEMS}

\section{A. The Axioms of QM revisited}

The well established standard axiomatic formulation of QM is based on four different classes of postulates (see 
e.g. [6]) dealing with description, measurements and evolution of a physical system. The first postulate concerns the "static" description of the system, asserting that to any physical system there is an associated Hilbert space whose dimension corresponds to the number of "degrees of freedom" of the physical system. If complete information about the physical system is available, the state of the system is represented by an element of this Hilbert space (pure state). Otherwise, the state is represented by a density matrix, i.e. a self-adjoint, positive semidefinite, normalized matrix acting on the Hilbert space. A second class of axioms of QM deals with the measurements and their outcomes. There is a third type of axiom dealing with indistinguishably of identical particles stating that bosons are represented by symmetric states in the corresponding Hilbert space while fermions by antisymmetric ones. These second and third type of axioms will not play any role in our considerations below. Finally, there exist a fourth type of axiom concerning the dynamics of a physical system. It states that the evolution (transformation from one state to another) of the system must be unitary. Thus, if a system evolves from a state $|\phi\rangle$ to a state $|\psi\rangle$, with $|\phi\rangle,|\psi\rangle \in \mathcal{H}$, then it must occur that $|\psi\rangle=U|\phi\rangle$, where $U$ is a unitary operator, i.e. $U^{\dagger} U=I$ ( $I$ being the identity operator on the Hilbert space $\mathcal{H}$ ). If a physical system evolves from a density matrix $\rho$ to a density matrix $\rho^{\prime}$, then it should happen that $\rho^{\prime}=U \rho U^{\dagger}$.

The logical structure of QM, and its relation to other fundamental principles of Nature has been for decades a subject of intensive research (see e.g. [2, 3, 7, 8, 9, 10, 11, 12, 13, 14, 15, 16, 17, 18]). In particular, the question whether the dynamical axiom can be derived from other assumptions has been addressed in e.g. 19]. In this paper, we give an alternative derivation to show that this dynamical axiom can be derived from requiring that the evolution preserves disorder (as quantified by von Neumann entropy), in the spirit of the second law of thermodynamics. Furthermore, we will also consider a very weak assumption of probabilistic linearity on the dynamics of the system. More precisely, we consider maps $L$ fulfilling that:

(i) the disorder of a physical system $\rho$ is preserved in the evolution of the system transforming $\rho$ to $L(\rho)$. The disorder of a physical system in a state $\rho$ is quantified by its von Neumann entropy $S(\rho)$, given by

$$
S(\rho)=-\operatorname{tr} \rho \log _{2} \rho .
$$

(ii) Probabilistic linearity: If two physical systems, described by states $\rho_{1}$ and $\rho_{2}$, evolve to $L\left(\rho_{1}\right)$ and $L\left(\rho_{2}\right)$, then a physical system, described by a probabilistic mixture $p \rho_{1}+(1-p) \rho_{2}(0 \leq p \leq 1)$ of $\rho_{1}$ and $\rho_{2}$, evolves to $p L\left(\rho_{1}\right)+(1-p) L\left(\rho_{2}\right)$.

These are the only assumptions that we make on the dynamics of a physical system. We adopt here the "static" description of the physical system from Quantum Mechanics. But, by itself, this static description does not have any consequences on the dynamics of the system. Then we show that the evolution of the physical system can be either unitary or anti-unitary [20]. The converse of this statement is of course known: Unitary and antiunitary evolutions preserve von Neumann entropy and can be defined to be probabilistically linear. As will be discussed later, anti-unitary operators cannot describe continuous transformations or better to say they cannot be continously deformed to unitary operations. Let us remark here that this result was previously obtained by A. Peres [19], showing that there is a close relation between dynamical evolutions that violate some fundamental axiom of QM, like unitarity, and those which are forbidden by the second law of thermodynamics. We provide here an alternative derivation of this result.

\section{The assumption of preservation of disorder}

Let us first briefly discuss the question of quantification of disorder. Under some quite general assumptions, the amount of disorder of a probability distribution $\left\{p_{i}\right\}$ can be shown to be the Shannon entropy $H\left(\left\{p_{i}\right\}\right)=-\sum_{i} p_{i} \log _{2} p_{i}$. This quantifies, on average, the amount of information an observer gathers (being equal to the disorder that was in the system) when she/he gets to know the result of a random variable which is distributed according to $\left\{p_{i}\right\}$. (See Ref. [22] for details.) The above observation, along with the fact that the eigenvalues of a density matrix $\rho$ are positive and sum up to unity (i.e. they can be interpreted as probabilities), led von Neumann to define disorder in quantum mechanical systems as $S(\rho)$, given by Eq. (1), for a physical system in a state $\rho$. Notice that density matrices can be interpreted as statistical mixtures of projectors onto elements of a Hilbert space $\mathcal{H}$. And $S(\rho)=-\sum_{i=1}^{n} p_{i} \log _{2} p_{i}$, where the $p_{i}$ are the eigenvalues of $\rho$, so that the von Neumann entropy of $\rho$ is the Shannon entropy of the eigenvalues of $\rho$. This is the only reason that we give for our choice of von Neumann entropy as the measure of disorder. For an axiomatic approach to derive the von Neumann entropy, as well as other reasons and advantages of this choice, see Ref. 23]. In passing, notice that the introduction of the von Neumann entropy does not require that the evolution is unitary, or anti-unitary [24]. Note also that the assumption of preservation of disorder is not in contradiction with results of statistical mechanics. This point is further discussed in Appendix A

\section{The assumption of probabilistic linearity}

We now move on to discuss the assumption in item (ii). As noted before, density matrices can be interpreted as statistical mixtures of projectors onto elements of a Hilbert space $\mathcal{H}$. The probabilistic, or statistical interpretation of density matrices imply that a statistical mixture of two density matrices is a legitimate density ma- 
trix, implying by itself a "probabilistic linearity" of the dynamics. This probabilistic linearity of density matrices really employs the probabilistic (statistical) description of states, and that each element of the ensemble (that is described by a density matrix) evolves independently. This notion of linearity is used e.g. in classical mechanics and classical electrodynamics, when we deal with probabilistic mixtures of states of a system. This is one of the reasons, why we say that item (ii) is a "weak" assumption. Note that the probabilistic linearity is qualitatively different than the linearity of the quantum formalism on superpositions of vectors on a Hilbert space, which we do not assume. Note also that in our case, the evolution characterized by the map $L$ transforms a density matrix $\rho$ into a matrix which, in general, is not normalized.

\section{B. Evolutions that preserve disorder and are probabilistically linear are unitary}

The only states that have zero entropy are the pure states (one eigenvalue equals unity and the others vanish). Therefore (by item (i)) every pure state has to be mapped again to a pure state. Consider two arbitrary different pure states $\left|\phi_{1}\right\rangle\left\langle\phi_{1}\right|$ and $\left|\phi_{2}\right\rangle\left\langle\phi_{2}\right|$. (The bras and kets used in this paper are normalized to unity.) Since $L$ inevitably transform them to pure states we can write

$$
\begin{aligned}
& L\left(\left|\phi_{1}\right\rangle\left\langle\phi_{1}\right|\right)=d_{1}\left|\psi_{1}\right\rangle\left\langle\psi_{1}\right|, \\
& L\left(\left|\phi_{2}\right\rangle\left\langle\phi_{2}\right|\right)=d_{2}\left|\psi_{2}\right\rangle\left\langle\psi_{2}\right| .
\end{aligned}
$$

Although $L$ does not have to be norm-preserving, $L$ must transform density matrices into matrices, which after normalization are density matrices. Therefore $d_{1}$ and $d_{2}$ must be both positive. Otherwise, a statistical mixture of the outputs in Eq. (2), will not be a positive semidefinite matrix.

Consider now the transformation that takes $\left|\phi_{1}\right\rangle$ to $\left|\psi_{1}\right\rangle$ and $\left|\phi_{2}\right\rangle$ to $\left|\psi_{2}\right\rangle$. Unless $\left|\phi_{1}\right\rangle$ is orthogonal to $\left|\phi_{2}\right\rangle$, and, since the choice is completely arbitrary, there must be a component of $\left|\phi_{2}\right\rangle$ that is parallel to $\left|\phi_{1}\right\rangle$, and a component that is orthogonal to $\left|\phi_{1}\right\rangle$. Thus, there is a $|\Phi\rangle$ orthogonal to $\left|\phi_{1}\right\rangle$ such that

$$
\left|\phi_{2}\right\rangle=\lambda_{1}\left|\phi_{1}\right\rangle+\lambda_{2}|\Phi\rangle \text {. }
$$

We can draw the phase of $\lambda_{2}$ into $|\Phi\rangle$, so that we have $\lambda_{2} \geq 0$. The same argument applies to the $\left|\psi_{i}\right\rangle$. In this way

$$
\left|\psi_{2}\right\rangle=\mu_{1}\left|\psi_{1}\right\rangle+\mu_{2}|\Psi\rangle,
$$

with $\mu_{2} \geq 0$, and $|\Psi\rangle$ orthogonal to $\left|\psi_{1}\right\rangle$. Note that $\left|\lambda_{1}\right|=\sqrt{1-\lambda_{2}^{2}}$ and $\left|\mu_{1}\right|=\sqrt{1-\mu_{2}^{2}}$. The $\left|\lambda_{i}\right|$ and $\left|\mu_{i}\right|$ are now uniquely defined and depend on the projectors $\left|\phi_{i}\right\rangle\left\langle\phi_{i}\right|$ and $\left|\psi_{i}\right\rangle\left\langle\psi_{i}\right|$. Note that $\lambda_{2}$ and $\mu_{2}$ cannot vanish.

Consider now the state

$$
\rho=p\left|\phi_{1}\right\rangle\left\langle\phi_{1}|+(1-p)| \phi_{2}\right\rangle\left\langle\phi_{2}\right|,
$$

with $0 \leq p \leq 1$. The matrix corresponding to $\rho$ in the orthonormal basis $\left\{\left|\phi_{1}\right\rangle,|\Phi\rangle\right\}$ is

$$
\left(\begin{array}{cc}
p+(1-p)\left|\lambda_{1}\right|^{2} & (1-p) \lambda_{1} \lambda_{2} \\
(1-p) \lambda_{1}^{*} \lambda_{2} & (1-p) \lambda_{2}^{2}
\end{array}\right),
$$

where the * denotes a complex conjugation. This matrix has the eigenvalues

$$
\begin{aligned}
& s_{1}=\frac{1}{2}\left(1-\sqrt{1-4 p \lambda_{2}^{2}+4 p^{2} \lambda_{2}^{2}}\right), \\
& s_{2}=\frac{1}{2}\left(1+\sqrt{1-4 p \lambda_{2}^{2}+4 p^{2} \lambda_{2}^{2}}\right) .
\end{aligned}
$$

Due to probabilistic linearity (item (ii)), $L$ maps $\rho$ onto

$$
L(\rho)=p d_{1}\left|\psi_{1}\right\rangle\left\langle\psi_{1}\left|+(1-p) d_{2}\right| \psi_{2}\right\rangle\left\langle\psi_{2}\right| .
$$

which has the eigenvalues

$$
t_{1}=\frac{1}{2}(\alpha-\beta), \quad t_{2}=\frac{1}{2}(\alpha+\beta),
$$

where

$$
\begin{aligned}
& \alpha=p d_{1}+d_{2}-p d_{2}, \\
& \beta=\sqrt{\left(p d_{1}+d_{2}-p d_{2}\right)^{2}+4 p d_{1} d_{2} \mu_{2}^{2}(p-1)} .
\end{aligned}
$$

For $2 \times 2$ matrices with unit trace, their von Neumann entropies can be equal if and only if they have the same eigenvalues. Since $L$ has to be entropy preserving, the ratio between the eigenvalues of $\rho$ and $L(\rho)$ must be the same, so that

$$
\text { either } \quad \frac{s_{1}}{s_{2}}=\frac{t_{1}}{t_{2}}, \quad \text { or } \quad \frac{s_{1}}{s_{2}}=\frac{t_{2}}{t_{1}} .
$$

A short calculation yields

$$
\mu_{2}=\frac{\left|p\left(d_{1}-d_{2}\right)+d_{2}\right| \lambda_{2}}{\sqrt{d_{1} d_{2}}} .
$$

However, $\mu_{2}$ was introduced in Eq. (4) and hence cannot depend on the mixing parameter $p$, which was introduced later on. Therefore we must have, for all $p$,

$$
\frac{\delta \mu_{2}}{\delta p}=0
$$

which implies that

$$
d_{1}=d_{2} .
$$

Hence, the two arbitrary pure states $\left|\phi_{1}\right\rangle$ and $\left|\phi_{2}\right\rangle$ are mapped onto pure states with the same length. Furthermore (using $d_{1}=d_{2}$ ) it follows that $\mu_{2}=\lambda_{2}$. But this implies that

$$
\left|\mu_{1}\right|=\left|\lambda_{1}\right| .
$$

In other words, the modulus of the scalar product is preserved in the evolution:

$$
\left|\left\langle\psi_{1} \mid \psi_{2}\right\rangle\right|=\left|\left\langle\phi_{1} \mid \phi_{2}\right\rangle\right| .
$$


The conservation of the modulus of the scalar product is, however, a very strong condition, since using the Wigner's theorem [25] (see Refs. 26, 27] in this regard), we obtain now that the transformation that induces

$$
\begin{aligned}
\left|\phi_{1}\right\rangle & \rightarrow\left|\psi_{1}\right\rangle, \\
\left|\phi_{2}\right\rangle & \rightarrow\left|\psi_{2}\right\rangle,
\end{aligned}
$$

is either linear and unitary or antilinear and anti-unitary. Here linearity means that if a transformation induces the transitions in Eq. (10), then the same transformation induces the transition $a\left|\phi_{1}\right\rangle+b\left|\phi_{2}\right\rangle \rightarrow a\left|\psi_{1}\right\rangle+b\left|\psi_{2}\right\rangle$. And antilinearity means that the obtained vector is $a^{*}\left|\psi_{1}\right\rangle+$ $b^{*}\left|\psi_{2}\right\rangle$.

Continuous transformations cannot be described by antilinear operators, as two consecutive antilinear operators act as a linear operator. We will disregard the option of anti-unitary operators for this reason. The evolution of our physical system is not given by Eq. (10), instead, it is given by Eq. (2). However, we have shown that $d_{1}=d_{2}$. Thus, the evolution on our physical system is unitary, up to a constant. As this constant is independent of the input state, it is irrelevant. Therefore we have reached our goal. The evolutions that respect the second law of thermodynamics and are probabilistically linear, are just the ones which are postulated in Quantum Mechanics: linear (on superpositions of vectors) and unitary.

\section{COMPOSITE QUANTUM SYSTEMS}

In this second part of the paper, we extend our study to bipartite (pure) quantum systems. We restrict our study to maps that are linear (on superpositions of pure states) and preserve entanglement. We will show that demanding qualitative preservation of entanglement, i.e. mapping separable states onto separable states and entangled states onto entangled states requires the map to be local or the product of a local map and the swap operator. The stricter requirement of preservation of some measure of entanglement requires the local maps to be essentially unitaries.

At this point, it is worth stressing the difference between the linearity postulates, assumed in this paper, for single and composite quantum systems. In Sec. [] we assumed a probabilistic linearity on the evolution maps, which is a rule for mixtures of density operators. In this section, on the other hand, evolution maps are assumed to be linear on superpositions of pure states. Henceforth, the latter version is simply referred to as "linear" maps.

\section{A. Formalism}

We restrict our discussion to bipartite systems, traditionally called Alice and Bob. The Hilbert space associated to the bipartite system is denoted by $\mathcal{H}_{A B}:=$ $\mathcal{H}_{A} \otimes \mathcal{H}_{B}$, where $\mathcal{H}_{A / B}$ are Hilbert spaces of dimension $n$, respectively $m$, over the field $\mathbb{C}$. We restrict ourselves to linear maps and allow the addition of local ancillas. The structure of the maps considered here is the following:

$$
L: \mathcal{H}_{A B} \mapsto \mathcal{H}_{A} \otimes \mathcal{H}_{A a} \otimes \mathcal{H}_{B} \otimes \mathcal{H}_{B a} .
$$

The above map $L$ could be decomposed into two different maps, the first one consisting only in adding local ancillas to the initial state, while the second one maps this state into the final one: $L: \mathcal{H} \hookrightarrow \mathcal{H} \otimes \mathcal{H}_{A a} \otimes \mathcal{H}_{B a} \stackrel{L^{\prime}}{\longrightarrow} \mathcal{H} \otimes \mathcal{H}_{A a} \otimes$ $\mathcal{H}_{B a}$. The first step is obviously entanglement preserving since the ancillas are in a product state. Thus for $L$ to be entanglement preserving, it is enough to demand $L^{\prime}$ to be entanglement preserving on the image of the inclusion. This means that $L^{\prime}$ acting on any state of the form $\left.|\psi\rangle \otimes\left|A_{a}\right\rangle \otimes B_{b}\right\rangle$ is entanglement preserving, but there might be more general states in $\mathcal{H} \otimes \mathcal{H}_{A a} \otimes \mathcal{H}_{B a}$ on which $L^{\prime}$ is not entanglement preserving.

By dimensional arguments $(\operatorname{dim}(\operatorname{range} L) \leq$ $\left.\operatorname{dim} \mathcal{H}_{A B}=n m\right)$ one can easily realise that there exist states in $\mathcal{H}_{A} \otimes \mathcal{H}_{A a} \otimes \mathcal{H}_{B} \otimes \mathcal{H}_{B a}$ which do not correspond to the image of $L$, i.e. there exist elements of $\mathcal{H}_{A} \otimes \mathcal{H}_{A a} \otimes \mathcal{H}_{B} \otimes \mathcal{H}_{B a}$ which are not reached by mapping the elements of $\mathcal{H}_{A B}$ with $L$. We can, therefore, restrict our investigation to a $\mathrm{nm}$-dimensional subspace $\mathcal{R} \subset \mathcal{H}_{A} \otimes \mathcal{H}_{A a} \otimes \mathcal{H}_{B} \otimes \mathcal{H}_{B a}$ into which all original states from $\mathcal{H}$ are mapped. Thus, mathematically we study linear, entanglement preserving maps of the form:

$$
L: \mathcal{H}_{A} \otimes \mathcal{H}_{B} \mapsto \mathcal{R} .
$$

We will show later that in fact $\mathcal{R}$ is isomorphic to $\mathcal{H}_{A B}$. In the most general case the map $L$ is not necessarily norm preserving. To keep track of this fact, as in Sect. I we use the "bra" and "ket" notation only for normalized states, i.e. $\langle\psi \mid \psi\rangle=1$. We shall explicitly write down any non-unit-length pre-factors (i.e. $c|\psi\rangle$ ). A map that decreases the norm of some states can be physically interpreted as a device that prepares the final state with a non-unit probability. In the remaining cases, the device prepares no system for the output. Norm-increasing maps are harder to interpret but we allow them for completeness. Finally, in our notation, if two states are parallel, i.e. if for a given $|\psi\rangle$ and $|\phi\rangle$, there exists a $c \in \mathbb{C}$ such that $|\psi\rangle=c|\phi\rangle$, we use the notation $|\psi\rangle \||\phi\rangle$. Non parallel states will be denoted by $|\psi\rangle \nVdash|\phi\rangle$.

\section{B. Entanglement preservation}

To characterize all maps that preserve entanglement, we have to first define this property (of entanglement). In the following, we distinguish between qualitative entanglement preservation and the stronger quantitative entanglement preservation. Qualitative entanglement preservation requires that separable states are mapped to separable states and entangled states are mapped to entangled states. This type of entanglement preservation 
leads to the fact that the map must be either local, or the product of a local map times the swap operator.

For a map to be quantitatively entanglement preserving, we should demand that there exists an entanglement measure $E[28,29]$, such that for all initial states $|\psi\rangle$ it holds $E(|\psi\rangle)=E(L(|\psi\rangle))$. It is known that there exists exactly one asymptotic measure of entanglement for pure normalized bipartite states, which is the von Neumann entropy of the reduced system of one of the two parties [30]. In the remainder of this paper, we will denote this quantity by $E(|\psi\rangle)$. Using naïvely the entropy function also on non-normalized states does not lead to a sensible entanglement measure. The entropy function evaluated on $|c|^{2}|a\rangle\langle a|$ (the reduced state of the pure product state $c|a b\rangle)$ gives $-|c|^{2} \log _{2}|c|^{2}$, which is 0 only for $|c| \in\{0,1\}$. There are, however, different ways to extend $E(|\psi\rangle)$ towards non-normalized states and in this paper we will deal with two possibilities: The first possibility is to normalize the state, i.e., to use the renormalized measure $E_{1}(c|\psi\rangle)=E(|\psi\rangle)$. This measure nullifies any physical significance that we may try to ascribe to a possible norm-change under the map. Alternatively, we may keep the norm as a multiplicative pre-factor, i.e., we use the probabilistic measure $E_{2}(c|\psi\rangle)=|c|^{2} E(|\psi\rangle)$. In the "failed map" interpretation of norm-loss, this measure is the average entanglement the device produces in the long run.

Of course, quantitative entanglement preservation implies qualitative entanglement preservation. But it is the quantitative preservation which allow us to demonstrate that such a map is, essentially, a local unitary. Before going into details, we review for completeness, some well established concepts such as the Schmidt decomposition for bipartite pure states and the swapping operator.

Bipartite systems always admit a Schmidtdecomposition, i.e. for every pure state $|\psi\rangle \in \mathcal{H}_{A B}$, there exists orthonormal bases $\left\{\left|a_{i}\right\rangle\right\}$ of $\mathcal{H}_{A}$ and $\left\{\left|b_{i}\right\rangle\right\}$ of $\mathcal{H}_{B}$, such that $|\psi\rangle=\sum_{i=1}^{r} \lambda_{i}\left|a_{i}\right\rangle \otimes\left|b_{i}\right\rangle$, with $\lambda \geq 0$ [31]. The $\lambda_{i}$ are denoted as the Schmidt coefficients and the $r$ as the Schmidt rank of the state $|\psi\rangle$. Hence the Schmidt rank is the minimal number of product states needed to decompose $|\psi\rangle$, and, by construction, is bounded by $r \leq \min (n, m)$.

Finally, we define here the swap operator as an special map that preserves entanglement. Swapping, in this paper, corresponds to exchange or relabeling the two subsystems and is mathematically a map $S: \mathcal{H}_{A} \otimes \mathcal{H}_{B} \mapsto$ $\mathcal{H}_{A^{\prime}} \otimes \mathcal{H}_{B^{\prime}}$, where $\mathcal{H}_{A^{\prime}}$ is isomorphic to $\mathcal{H}_{B}$ and $\mathcal{H}_{B^{\prime}}$ is isomorphic to $\mathcal{H}_{A}$. For a given orthonormal basis $\left\{\left|\mathbf{1}_{A}\right\rangle, \ldots,\left|\mathbf{n}_{A}\right\rangle\right\}$ of $\mathcal{H}_{A}$ and $\left\{\left|\mathbf{1}_{B}\right\rangle, \ldots,\left|\mathbf{m}_{B}\right\rangle\right\}$ of $\mathcal{H}_{B}$, the isomorphisms immediately lead to orthonormal bases $\left\{\left|\mathbf{1}_{A^{\prime}}\right\rangle, \ldots,\left|\mathbf{m}_{A^{\prime}}\right\rangle\right\}$ of $\mathcal{H}_{A^{\prime}}$ and $\left\{\left|\mathbf{1}_{B^{\prime}}\right\rangle, \ldots,\left|\mathbf{n}_{B^{\prime}}\right\rangle\right\}$ of $\mathcal{H}_{B^{\prime}}$. We define the swap operator as

$$
\hat{S}:\left|\mathbf{i}_{A}\right\rangle \otimes\left|\mathbf{j}_{B}\right\rangle \mapsto\left|\mathbf{j}_{A^{\prime}}\right\rangle \otimes\left|\mathbf{i}_{B^{\prime}}\right\rangle .
$$

Of course, a different isomorphism between $\mathcal{H}_{A / B}$ and $\mathcal{H}_{A^{\prime} / B^{\prime}}$, that identifies the bases $\left\{\left|\mathbf{i}_{A / B}\right\rangle\right\}$ with different orthonormal bases $\left\{\left|\tilde{\mathbf{i}}_{A^{\prime} / B^{\prime}}\right\rangle\right\}$ leads to a different swap operator. But since all local orthonormal bases are connected by local unitaries, all swap operators are also interconnected by local unitaries, that is, up to local unitaries, the swap operator is unique. Note here that the swap operator considered in this paper is different from the usual swap operator considered in the literature, which also allows exchanging a subspace of the Hilbert space $\mathcal{H}_{A}$ with that of $\mathcal{H}_{B}$. Such a general swap operator can of course increase (or decrease) the entanglement between $A$ and $B$ : Simply consider exchanging $A^{\prime \prime}$ with $B^{\prime \prime}$ (and back) for the state $\left|\Psi^{-}\right\rangle_{A^{\prime} A^{\prime \prime}}|0\rangle_{B^{\prime}}|0\rangle_{B^{\prime \prime}}$ in the $A^{\prime} A^{\prime \prime}: B^{\prime} B^{\prime \prime}$ bipartite cut, where $\left|\Psi^{-}\right\rangle=\frac{1}{\sqrt{2}}(|01\rangle-|10\rangle)$.

\section{Qualitative entanglement preservation}

Qualitative entanglement preservation is equivalent to the demand that the set of states with Schmidt rank $r=1$ (product states) and the set of states with Schmidt rank $r>1$ (entangled states) are both invariant under application of the map $L$.

Fact 1 A qualitatively entanglement preserving linear map has to be of full rank.

Proof: Suppose that the kernel of a linear qualitatively entanglement preserving map $L$ is not empty. Assume that there exist a product vector $\left|a_{1}, b_{1}\right\rangle$ which belongs to the kernel of $L$, i.e. $L\left|a_{1}, b_{1}\right\rangle=0$. Let us choose a second product vector $\left|a_{2}, b_{2}\right\rangle$, for which $\left|a_{2}\right\rangle,\left|b_{2}\right\rangle$ are not parallel to $\left|a_{1}\right\rangle,\left|b_{1}\right\rangle$ respectively. Any (nontrivial) combination of these two product states $\left|\psi_{2}\right\rangle$, is an entangled state of Schmidt rank 2. But unless $\left|a_{2}, b_{2}\right\rangle$ is also in the kernel, $L$ cannot be entanglement preserving. Thus, if there exist one product state in the kernel, then one can find a basis of product states all of them belonging to the kernel of $L$, and hence $L$ is the zero map. It is enough to show now that there exit no entangled state of Schmidt rank 2 in the kernel of $L$. Assume that there exist one entangled state of rank $2\left|\phi_{2}^{+}\right\rangle=\lambda_{1}\left|e_{1}, f_{1}\right\rangle+\lambda_{2}\left|e_{2}, f_{2}\right\rangle$, written in the Schmidt decomposition with $\lambda_{i}>0$ in the kernel of $L$, i.e. $L\left(\left|\phi_{2}^{+}\right\rangle\right)=0$. We construct $\left|\phi_{2}^{-}\right\rangle=\lambda_{1}\left|e_{1}, f_{1}\right\rangle-\lambda_{2}\left|e_{2}, f_{2}\right\rangle$ which is also of rank 2 . However, $(1 / 2)\left(\left|\phi_{2}^{+}\right\rangle+\left|\phi_{2}^{-}\right\rangle\right)=\lambda_{1}\left|e_{1}, f_{1}\right\rangle$ and therefore $L\left(\left|\phi_{2}^{-}\right\rangle\right)$must be of rank 1 , which cannot be since $L$ is entanglement preserving map. Therefore, there exist not entangled state of rank $r=2$ in the Kernel of $L$. It is now obvious to see that if the kernel of $L$ does not contain any product vector and any Schmidt rank 2 vector, then it cannot contain any vector of Schmidt rank $r>2$. Therefore, we have shown that if $L$ is a non trivial entanglement preserving map, its kernel is empty and $L$ is of full rank.

We observe that $L$ maps all product states to multiples of product states. Therefore, $L$ cannot increase the Schmidt rank of a pure state: If $|\phi\rangle=\sum_{i=1}^{r} \lambda_{i}\left|a_{i} b_{i}\right\rangle$, then $L|\phi\rangle=\sum_{i=1}^{r} \lambda_{i} L\left|a_{i} b_{i}\right\rangle=\sum_{i=1}^{r} \lambda_{i} c_{i}\left|a_{i}^{\prime} b_{i}^{\prime}\right\rangle$ is a superposition of at most $r$ product terms, and the Schmidt rank of $L|\phi\rangle$ cannot be larger than $r$. Furthermore, we know 
from Fact 1 that $L$ is invertible, that is, for each $L$ that is entanglement preserving, there exists an $L^{-1}$ with the property that $L^{-1} L|\phi\rangle=|\phi\rangle$. Thus $L^{-1}$ is also a linear qualitatively entanglement preserving map. Thus neither $L$ or $L^{-1}$ can increase the Schmidt rank. So, starting from the weak demand of qualitative entanglement preservation we have proved

Fact 2 The Schmidt rank is invariant under the application of any linear, qualitatively entanglement preserving map $L$.

We are now ready to state our main result regarding qualitative entanglement preservation.

Result 1 Every linear map that is qualitatively entanglement preserving has to be either local or a local operator times the swap operator.

The first part of our proof of this result is slightly technical, and proceeds by looking at some specific states and their image under an entanglement preserving map. To do this, we begin by choosing an orthonormal basis $\left\{\left|\mathbf{1}_{A}\right\rangle, \ldots,\left|\mathbf{n}_{A}\right\rangle\right\}$ of Alice's Hilbert space $\mathcal{H}_{A}$, and an orthonormal basis $\left\{\left|\mathbf{1}_{B}\right\rangle, \ldots,\left|\mathbf{m}_{B}\right\rangle\right\}$ of Bob's Hilbert space $\mathcal{H}_{B}$. Due to the preservation of separability, we know that $L$ acts on an element of the product basis $\left|\mathbf{i}_{A}, \mathbf{j}_{B}\right\rangle$ as:

$$
\left|\mathbf{i}_{A}, \mathbf{j}_{B}\right\rangle \stackrel{L}{\rightarrow} c_{i, j}\left|d_{i, j}\right\rangle \otimes\left|e_{i, j}\right\rangle,
$$

where the length $c_{i, j}$ and both tensor factors can a priori depend on both input factors $\left|\mathbf{i}_{A}\right\rangle$ and $\left|\mathbf{j}_{B}\right\rangle$. Since $L$ is linear, this evaluation on a basis fixes $L$ completely. Now we start by applying $L$ to a state of Schmidt rank 2. Let $i \neq k$ and $j \neq l$ and we look at the transformation

$$
\left|\mathbf{i}_{A} \mathbf{j}_{B}\right\rangle+\left|\mathbf{1}_{A} \mathbf{k}_{B}\right\rangle \stackrel{L}{\longrightarrow} c_{i, j}\left|d_{i, j}\right\rangle \otimes\left|e_{i, j}\right\rangle+c_{k, l}\left|d_{k, l}\right\rangle \otimes\left|e_{k, l}\right\rangle .
$$

As the right hand is not a product state, we must have $\left|d_{i, j}\right\rangle \nVdash\left|d_{k, l}\right\rangle$ and $\left|e_{i, j}\right\rangle \nVdash\left|e_{k, l}\right\rangle$, i.e., when both indices differ, the image vectors cannot be parallel. On the other hand, consider the following application of the map to a product vector:

$$
\begin{array}{r}
\left|\mathbf{1}_{A} \mathbf{1}_{B}\right\rangle+\left|\mathbf{1}_{A} \mathbf{2}_{B}\right\rangle=\left|\mathbf{1}_{A}\right\rangle \otimes\left[\left|\mathbf{1}_{B}\right\rangle+\left|\mathbf{2}_{B}\right\rangle\right] \\
\stackrel{L}{\rightarrow} c_{1,1}\left|d_{1,1}\right\rangle \otimes\left|e_{1,1}\right\rangle+c_{1,2}\left|d_{1,2}\right\rangle \otimes\left|e_{1,2}\right\rangle
\end{array}
$$

It is clear that either (i) $\left|d_{1,2}\right\rangle \|\left|d_{1,1}\right\rangle$, or (ii) $\left|e_{1,2}\right\rangle \|$ $\left|e_{1,1}\right\rangle$.

It turns out that in case (i), $\left|d_{i, j}\right\rangle \|\left|d_{i, i}\right\rangle$ and $\left|e_{i, j}\right\rangle \|$ $\left|e_{j, j}\right\rangle$, while in case (ii), it is the other way around: the $|d\rangle$ image vectors only depend on the second index, while the $|e\rangle$ image vectors only depend on the first index.

To prove this, assume that we know that $\left|d_{i, j}\right\rangle \|\left|d_{i, l}\right\rangle$ for $j \neq l$ and we look at

$$
\begin{aligned}
& \left|\mathbf{i}_{A} \mathbf{j}_{B}\right\rangle+\left|\mathbf{k}_{A} \mathbf{j}_{B}\right\rangle=\left[\left|\mathbf{i}_{A}\right\rangle+\left|\mathbf{k}_{A}\right\rangle\right] \otimes\left|\mathbf{j}_{B}\right\rangle \\
& \stackrel{L}{\longrightarrow} c_{i, j}\left|d_{i, j}\right\rangle \otimes\left|e_{i, j}\right\rangle+c_{k, j}\left|d_{k, j}\right\rangle \otimes\left|e_{k, j}\right\rangle,
\end{aligned}
$$

for some $k \neq i$. On the right hand side, the $d$-factors cannot be parallel, since $\left|d_{i, j}\right\rangle \|\left|d_{i, l}\right\rangle$ and $\left|d_{i, l}\right\rangle \nVdash\left|d_{k, j}\right\rangle$ as we pointed out before since they differ in both indices. Therefore we find that

$$
\exists l \neq j:\left|d_{i, j}\right\rangle \|\left|d_{i, l}\right\rangle \quad \Rightarrow \quad\left|e_{i, j}\right\rangle \|\left|e_{k, j}\right\rangle \quad \forall k,
$$

and in an analogous way we can prove that

$$
\exists k \neq i:\left|e_{i, j}\right\rangle \|\left|e_{k, j}\right\rangle \quad \Rightarrow \quad\left|d_{i, j}\right\rangle \|\left|d_{i, l}\right\rangle \quad \forall l .
$$

Using $\left|d_{1,2}\right\rangle \|\left|d_{1,1}\right\rangle$ as the starting point, it is now easy to show the desired result for case (i):

$$
\begin{aligned}
\left|d_{1,2}\right\rangle \|\left|d_{1,1}\right\rangle & \Rightarrow \quad\left|e_{i, 1}\right\rangle \|\left|e_{1,1}\right\rangle \quad \forall i \\
\Rightarrow \quad\left|d_{i, j}\right\rangle \|\left|d_{i, i}\right\rangle \quad \forall i, j & \Rightarrow\left|e_{i, j}\right\rangle \|\left|e_{j, j}\right\rangle \quad \forall i, j
\end{aligned}
$$

which means that

$$
\left|\mathbf{i}_{A} \mathbf{j}_{B}\right\rangle \stackrel{L}{\rightarrow} c_{i, j}\left|d_{i, j}\right\rangle \otimes\left|e_{i, j}\right\rangle=\tilde{c}_{i, j}\left|d_{i, i}\right\rangle\left|e_{j, j}\right\rangle,
$$

where $\tilde{c}_{i, j}$ may contain extra phase factors as compared to $c_{i, j}$. Therefore we have obtained a decomposition of $L$ in the form

$$
L=L_{A} \otimes L_{B} P,
$$

where $L_{A / B}: \mathcal{H}_{A / B} \mapsto \mathcal{H}_{A / B}$, with $L_{A}\left|\mathbf{i}_{A}\right\rangle=\left|d_{i, i}\right\rangle$ and $L_{B}\left|\mathbf{j}_{B}\right\rangle=\left|e_{j, j}\right\rangle$ and $P$ is a global phase/length with $P$ : $\mathcal{H}_{A B} \mapsto \mathcal{H}_{A B}$ with $P\left|\mathbf{i}_{A} \mathbf{j}_{B}\right\rangle \mapsto \tilde{c}_{i, j}\left|\mathbf{i}_{A} \mathbf{j}_{B}\right\rangle$. Obviously, the map $P$ is diagonal in the chosen product basis, but since $L_{A} \otimes L_{B}$ is local, it is also clear that $P$ must map all product vectors to product vectors in order to fulfill the preservation of separability. For case (ii), Eqs. (18) and (19) must be replaced by their equivalents with $d$ and $e$ swapped. Instead of Eq. (21), we then obtain

$$
\left|\mathbf{i}_{A} \mathbf{j}_{B}\right\rangle \stackrel{L}{\rightarrow} c_{i, j}\left|d_{i, j}\right\rangle \otimes\left|e_{i, j}\right\rangle=\tilde{c}_{i, j}\left|d_{j, j}\right\rangle\left|e_{i, i}\right\rangle .
$$

Since the set $\left\{\left|\mathbf{i}_{A} \mathbf{j}_{B}\right\rangle\right\}$ spans $\mathcal{H}_{A B}$, and $L$ is of full rank, the image of these vectors, i.e. the set $\left\{\left|d_{j, j}\right\rangle\left|e_{i, j}\right\rangle\right\}$, has also to span the whole space $\mathcal{R}$, which is $n m$ dimensional. So both sets $\left\{\left|d_{j, j}\right\rangle\right\}$ and $\left\{\left|e_{i, i}\right\rangle\right\}$ have to be linearly independent, and span a $m$ dimensional Hilbert space $\mathcal{H}_{A^{\prime}}$, respectively a $n$ dimensional Hilbert space $\mathcal{H}_{B^{\prime}}$, so that

$$
\mathcal{R}=\mathcal{H}_{A^{\prime}} \otimes \mathcal{H}_{B^{\prime}}
$$

In this case, the combined map $S \circ L$, where $S$ is the swap operator, maps from the space $\mathcal{H}_{A B}$ into itself (or more specifically in a space isomorphic to $\mathcal{H}_{A B}$ ), and can be decomposed as

$$
S \circ L=L_{A} \otimes L_{B} P,
$$

where $L_{A}\left|\mathbf{i}_{\mathbf{A}}\right\rangle=\left|e_{i, i}\right\rangle, L_{B}\left|\mathbf{j}_{\mathbf{B}}\right\rangle=\left|d_{j, j}\right\rangle$ and $P\left|\mathbf{i}_{\mathbf{A}} \mathbf{j}_{\mathbf{B}}\right\rangle=$ $\tilde{c}_{i, j}\left|\mathbf{i}_{\mathbf{A}} \mathbf{j}_{\mathbf{B}}\right\rangle$. As in the first case, $P$ maps all product vectors to product vectors.

To complete the proof of Result 1 we now only need 
Fact 3 Every linear map that takes all product states to product states, is of full rank, and is diagonal in some product basis, has to be local.

To prove this, we proceed as follows. Let $P$ be of diagonal form in the product basis $\left|a_{i} b_{j}\right\rangle$, that is

$$
P\left|a_{i} b_{j}\right\rangle=\lambda_{i j}\left|a_{i} b_{j}\right\rangle
$$

We know that $P$ maps the product state $\left(\sum_{i} \alpha_{i}\left|a_{i}\right\rangle\right) \otimes$ $\left(\sum_{j} \beta_{j}\left|b_{j}\right\rangle\right.$ ) (where we take the $\alpha_{i} \neq 0 \neq \beta_{j}$ as arbitrary but fixed) to a product state, which can in general be written as $\sum_{i, j} x_{i} y_{j}\left|a_{i} b_{j}\right\rangle$. This can only hold if for all $i$ and $j$

$$
x_{i} y_{j}=\lambda_{i j} \alpha_{i} \beta_{j}
$$

Since $P$ is of full rank, all $\lambda_{i j} \neq 0$, and thus, it also has to hold that $x_{i} y_{j} \neq 0$ and we can write

$$
\frac{y_{j}}{y_{k}}=\frac{\lambda_{i j}}{\lambda_{i k}} \frac{\beta_{j}}{\beta_{k}} .
$$

The left hand side of eq (28) does not depend on $i$, so the fraction $\chi_{j k}:=\frac{\lambda_{i j}}{\lambda_{i k}}$ does neither. This gives us a separation of the form

$$
\lambda_{i j}=\lambda_{i 0} \chi_{j 0}=: \mu_{i} \nu_{j}
$$

Therefore $P=P_{A} \otimes P_{B}$ as stated above, where $P_{A}\left|a_{i}\right\rangle=$ $\mu_{i}\left|a_{i}\right\rangle$ and $P_{B}\left|b_{j}\right\rangle=\nu_{j}\left|b_{j}\right\rangle$.

\section{Quantitative entanglement preservation}

In the previous section, we only required a qualitative preservation of entanglement. In this section, we will discuss the quantitative preservation of entanglement. As stated in Sec. IIB a quantitative preservation of entanglement depends on the extension of the von Neumann entropy of the reduced system that is used. However, for both of the two previously defined entanglement measures, $E_{1}$ and $E_{2}$, we have that

Result 2 Every local and linear map $L$ that is quantitatively entanglement preserving, has to be a multiple of a local unitary.

We have proved in the previous section that a qualitatively entanglement preserving map is a local map or a local map after the application of the swap operator. Every quantitatively entanglement preserving map is also a qualitatively entanglement preserving one, so we know that $L=L_{A} \otimes L_{B}$. Due to the singular value decomposition of $L_{A}$ and $L_{B}$ we have

$$
L_{A}=V_{A} D_{A} U_{A}, \quad L_{B}=V_{B} D_{B} U_{B}
$$

where $U_{A}, U_{B}, V_{A}, V_{B}$ are unitary matrices, $D_{A}$ is a diagonal matrix in the basis $\left\{\left|\mathbf{1}_{A}\right\rangle, \ldots,\left|\mathbf{n}_{A}\right\rangle\right\}$ with the diagonal values $\lambda_{1} \geq \lambda_{2} \geq \ldots \geq \lambda_{n}>0$, and $D_{B}$ is a diagonal matrix in the basis $\left.\left\{\mathbf{1}_{B}\right\rangle, \ldots,\left|\mathbf{m}_{B}\right\rangle\right\}$ with the diagonal values $\mu_{1} \geq \mu_{2} \geq \ldots \geq \mu_{m}>0$. We define four further sets of orthonormal bases by

$$
\begin{aligned}
\left|a_{i}\right\rangle:=U_{A}^{-1}\left|i_{A}\right\rangle, & \left|b_{i}\right\rangle:=U_{B}^{-1}\left|i_{B}\right\rangle, \\
\left|e_{i}\right\rangle:=V_{A}\left|i_{A}\right\rangle, & \left|f_{i}\right\rangle:=V_{B}\left|i_{B}\right\rangle .
\end{aligned}
$$

For the respective proofs we will deal with a special set of states defined as

$$
\left.|\psi(c)\rangle=c\left|a_{1} b_{1}\right\rangle+\sqrt{1-|c|^{2}}\left|a_{n} b_{m}\right\rangle\right),
$$

where $c \in[0,1]$. These states transform under $L$ into

$$
L|\psi(c)\rangle=c \lambda_{1} \mu_{1}\left|e_{1} f_{1}\right\rangle+\lambda_{n} \mu_{m} \sqrt{1-|c|^{2}}\left|e_{n} f_{n}\right\rangle .
$$

Note that Eq. (33) expresses $L|\psi\rangle$ already in its Schmidt decomposition.

The way to proceed now depends on the entanglement measure we choose.

\section{The renormalized measure of entanglement $E_{1}$}

In this subsection we will use the entanglement measure $\left.E_{1}(\kappa|\psi\rangle):=E(\psi\rangle\right)$. Let us look at the state $\left|\psi\left(c_{1}\right)\right\rangle$ of (32) with $c_{1}=1 / \sqrt{2}$. This is obviously a maximally entangled state with Schmidt rank 2: $E_{1}\left(\left|\psi\left(c_{1}\right)\right\rangle\right)=$ $E\left(\left|\psi\left(c_{1}\right)\right\rangle\right)=1$. The state (33) has this entanglement value only if $\lambda_{1} \mu_{1}=\lambda_{n} \mu_{m}$, which fixes $\lambda_{n}=\lambda_{1}$ and $\mu_{m}=\mu_{1}$. (We remember that the $\mu$ 's and $\nu$ 's are ordered.) So $D_{A}=\lambda_{1} \mathbf{1}_{A}$ and $D_{B}=\mu_{1} \mathbf{1}_{B}$, which then leaves us with

$$
L_{A}=\lambda_{1} V_{A} U_{A}, \quad L_{B}=\mu_{1} V_{B} U_{B},
$$

which are multiples of products of unitary matrices. This concludes the proof of Result 2 for the renormalized entanglement measure.

\section{The probabilistic measure of entanglement $E_{2}$}

We will now study the entanglement measure $E_{2}(c|\psi\rangle)=|c|^{2} E(|\psi\rangle)$. Let us start with the physically most reasonable case, namely $\lambda_{1} \mu_{1} \leq 1$. Assume now that we actually have $\lambda_{n} \mu_{m}<1$. Then the entanglement of the maximally entangled state $\left|\psi\left(\frac{1}{\sqrt{2}}\right)\right\rangle=\frac{1}{\sqrt{2}}\left(\left|a_{1} b_{1}\right\rangle+\right.$ $\left.\left|a_{n} b_{m}\right\rangle\right)$ cannot be preserved, since this state would be mapped to the multiple of a state that is at most as entangled as the input state, but the length (or the probability of getting this state) would be $\sqrt{\frac{\lambda_{1} \mu_{1}^{2}}{2}+\frac{\lambda_{n} \mu_{m}^{2}}{2}}<1$, leading to an even smaller actual value of the probabilistic measure of entanglement. Therefore we would have in this case $\lambda_{1}=\lambda_{n}=1=\mu_{1}=\mu_{m}$, and $L$ is unitary.

Since $L$ is of full rank, it is invertible and $L^{-1}$ is also entanglement preserving, so that similar arguments can 
be used to deal with the case $\lambda_{n} \mu_{m} \geq 1$. The only remaining case is then $\lambda_{1} \mu_{1} \geq 1 \geq \lambda_{n} \mu_{m}$, and we deal with that in Appendix $\mathrm{B}$ since it is rather technical, and moreover this case involves the rather unreasonable argument of the increase in norm for some states.

\section{CONCLUSION}

Summarizing, we have investigated the properties of maps that preserve either entropy in single quantum systems or entanglement in composite quantum systems. The first part of the paper demonstrates that the evolutions that respect the second law of thermodynamics and are probabilistically linear, are just the ones which are postulated in Quantum Mechanics: linear (on superpositions of vectors) and unitary. In the second part of the paper we have shown, that if from a linear map $L$ (linear on superpositions of vectors), we additionally demand preservation of entanglement in a very reasonable form (precisely that product states are mapped to product states and entangled states to entangled ones), then one immediately obtains preservation of the Schmidt rank. From this fact we have concluded that every reasonable entanglement preserving map has to be either local or local after application of a swap operation. In a further step, we have studied the consequences that two special extensions of the unique asymptotic entanglement measure for pure bipartite states, induce on the two local parts of $L$. We were able to show that for the probabilistic measure of entanglement $E_{2}$, the linear map $L$ is in fact a local unitary, or a local unitary after the application of the swap. And in the case of the renormalized measure of entanglement $E_{1}$, the map is either a multiple of a local unitary, or a multiple of a local unitary after the application of the swap. But, in this case (of $E_{1}$ ), we can drop the factor, since we renormalize the outcome, for the evaluation of the entanglement. Furthermore, we have shown along the way, that the addition of the local ancillas does not increase the set of allowed maps since the space mapped into has to remain isomorphic to the composite Hilbert space $\mathcal{H}_{A B}$.

It is tempting to think of our results in the following way: entropy is, of course, preserved in unitary dynamics. However, for systems whose dynamics is not assumed to be quantum, entropy preservation can be seen as the second law of thermodynamics (for reversible processes). On the other hand, for composite systems, it is clear that entanglement is preserved by local unitary dynamics and (complete) exchange of systems. However, for composite systems on which we do not put any dynamical assumption, preservation of entanglement has been proposed as an equivalent second law of thermodynamics for composite systems (see e.g. 38]). The premises we use could, therefore, be regarded as second laws for single and composite systems plus some forms of linearity on the evolution. And under these assumptions we have shown that the evolution must be unitary.

\section{Acknowledgments}

We would like to thank William K. Wootters for illuminating correspondence, Dagmar Bruß and Klaus Dietz for discussions, and the participants of the A2 Consortium. We acknowledge support from the DFG (SFB 407, SPP 1078 "Quantum Information Processing" and the European Graduate College "Interference and Quantum Applications", 432POL), the Alexander von Humboldt Foundation, the EC program QUPRODIS, and the ESF Program QUDEDIS.

\section{APPENDIX A}

In this Appendix, we argue that the assumption of preservation of disorder in reversible processes (item(i) of IA can coexist with statistical mechanics, in particular with the Boltzmann $H$ theorem.

Suppose that there are $N$ classical particles of a gas in a box, such that the probability density at position $\vec{r}$, momentum $\vec{p}$, and time $t$, is given by the distribution function $f(\vec{r}, \vec{p}, t)$. Then if the particles follow classical mechanical equations, the quantity

$$
H(t)=-\int d \vec{r} d \vec{p} f(\vec{r}, \vec{p}, t) \log _{2} f(\vec{r}, \vec{p}, t)
$$

can be shown to be an increasing function (under certain assumptions). This is the Boltzmann $H$ theorem 32, 33]. Identifying $H(t)$ with the disorder of the system, it may seem that the Boltzmann $H$ theorem is against the hypothesis of preservation of disorder. However the $H$ theorem is true in the limit of "molecular chaos" [34], which means that in a volume element $d \vec{r}$ around the position $\vec{r}$, the probability of finding two particles with momenta $\vec{p}_{1}$ and $\vec{p}_{2}$ are independent, at any instant of time $t$, so that the probability of finding them simultaneously is the product $f\left(\vec{r}, \vec{p}_{1}, t\right) f\left(\vec{r}, \vec{p}_{2}, t\right)$ of the individual probabilities. It is known that under such an assumption of factorization, an otherwise closed system turns into an open system, leading to irreversibility in the process (see e.g. [35]).

Our considerations are however for reversible processes, and so the $H$ theorem is not relevant for our purposes. Remaining still within the realm of classical mechanics, the Liouville density $D$ of a system of $N$ (classical) particles in the $6 N$-dimensional phase space can be used to define the disorder of the $N$ particle gas as the Gibbs entropy

$$
H_{G}(t)=-\int d \Gamma D \log _{2} D
$$

where $d \Gamma$ is an element of the phase space. Using Liouville's theorem (stating that $D$ is a constant of motion) [36], it is straightforward to show that the Gibbs entropy is a constant of (classical) motion.

Similar considerations hold in the quantum domain. It is known [1] (see also [37]) that a quantum system 
approaches its equilibrium state through multiple collisions with other systems in equilibrium. In the process of reaching this equilibrium, the von Neumann entropy of the system increases, compatible with the average energy of the system. In the absence of any such interactions with other external systems, the von Neumann entropy of the system remains constant, as a consequence of the unitarity of its evolution. For an interacting system, the transformation of the system along with the heat bath is again unitary, and hence the entropy of the system plus the heat bath is again preserved. However, when we consider the system only, we see an increase of entropy. Such a system is open, and leads, quite generally, to irreversibility.

\section{APPENDIX B}

In this appendix we close the gap in the argument concerning the probabilistic measure of entanglement. We have to deal with the case when for the $\lambda_{1} \mu_{1}$ and $\lambda_{n} \mu_{m}$ of Eq. (33), the inequality $\lambda_{1} \mu_{1} \geq 1 \geq \lambda_{n} \mu_{m}$ holds. Due to continuity of the lengths of the vectors, there exists a $c_{2}$ such that the the normalized vector $\left|\psi\left(c_{2}\right)\right\rangle$ (of the form in Eq. (32) is mapped by $L$ to a normalized vector (in the form in Eq. (33)), i.e.

$$
\begin{aligned}
\left|\psi\left(c_{2}\right)\right\rangle: & =c_{2}\left|a_{1} b_{1}\right\rangle+\sqrt{1-c_{2}^{2}}\left|a_{n} b_{m}\right\rangle \\
& \stackrel{L}{\longrightarrow} l\left|x_{1} y_{1}\right\rangle+\sqrt{1-l^{2}}\left|x_{n} y_{m}\right\rangle .
\end{aligned}
$$

Due to preservation of entanglement by $L$ the Schmidt values of the two vectors have to be preserved, and we have that either $c_{2}^{2} \lambda_{1}^{2} \mu_{1}^{2}=c_{2}^{2}$ and $\left(1-c_{2}^{2}\right) \lambda_{n} \mu_{m}=(1-$ $c_{2}^{2}$ ), which immediately results in $\lambda_{1} \mu_{1}=1=\lambda_{n} \mu_{m}$, or $c_{2}^{2} \lambda_{1}^{2} \mu_{1}^{2}=1-c_{2}^{2}$ and $\left(1-c_{2}^{2}\right) \lambda_{n} \mu_{m}=c_{2}^{2}$. The last two equations can be combined to

$$
\lambda_{1} \mu_{1} \lambda_{n} \mu_{m}=1 .
$$

Now, since $L$ is of full rank, every vector is in the range of $L$. So there exists a $c_{3}$ such that

$$
\begin{array}{r}
\left|\psi\left(c_{3}\right)\right\rangle:=c_{3}\left|a_{1} b_{1}\right\rangle+\sqrt{1-c_{3}^{2}}\left|a_{n} b_{m}\right\rangle \\
\quad \stackrel{L}{\rightarrow} \sqrt{E\left(c_{3}\right)} \frac{1}{\sqrt{2}}\left(\left|x_{1} y_{1}\right\rangle+\left|x_{n} y_{m}\right\rangle\right),
\end{array}
$$

where $E\left(c_{3}\right)$ is the entanglement of $\left|\psi\left(c_{3}\right)\right\rangle$, which only depends on $c_{3}$. We therefore have the requirement

$$
\lambda_{1} \mu_{1}=\sqrt{\frac{E\left(c_{3}\right)}{2 c_{3}^{2}}}, \quad \lambda_{n} \mu_{m}=\sqrt{\frac{E\left(c_{3}\right)}{2\left(1-c_{3}^{2}\right)}} .
$$

Using the fact that the product of the two terms has to equal 1 , as shown before, we have that

$$
\frac{E\left(c_{3}\right)}{\sqrt{c_{3}^{2}\left(1-c_{3}^{2}\right)}}=2
$$

which can easily be seen to have the only positive solution $c_{3}=\frac{1}{\sqrt{2}}$. But this corresponds to the case where it is a maximally entangled state that is taken to a maximally entangled state, and $\lambda_{1} \mu_{1}=1$ as well as $\lambda_{n} \mu_{m}=1$. So one can take $\lambda_{i}=1$ and $\mu_{j}=1$, which shows by Eq. (34) that $L_{A}$ and $L_{B}$ are actually unitary maps. So $L$ is the product of two local unitaries, and not the multiple thereof, like in the case of the renormalized measure of entanglement.
[1] J.S. Bell, Speakable and Unspeakable in Quantum Mechanics (Cambridge University Press, Cambridge, 1987); L.E: Ballentine, editor, Foundations of Quantum Mechanics since the Bell Inequalities, Amer. Assoc, Phys. Teachers, College Park (1988), and references therein.

[2] A. Einstein, B. Podolsky, and N. Rosen, Phys. Rev. 47, 777 (1935).

[3] J.S. Bell, Physics 1, 195 (1964). J.S. Bell, Rev. Mod. Phys. 38, 447 (1966).

[4] E. Santos, Phys. Rev. Lett. 66, 1388 (1991); E. Santos, Phys. Rev. A 46, 3646 (1992).

[5] P.M. Pearle, Phys. Rev. D 2, 1418 (1970); J.F. Clauser and M.A. Horne, Phys. Rev. D 10, 526 (1974); P.G. Kwiat, P.H. Eberhard, A.M. Steinberg, and R.Y. Chiao, Phys. Rev. A 49, 3209 (1994); N. Gisin and B. Gisin, Phys. Lett. A 260, 323 (1999); S. Massar, S. Pironio, J. Roland, and B. Gisin, Phys. Rev. A 66, 052112 (2002); R. Garcia-Patron, J. Fiurásek, N.J. Cerf, J. Wenger, R. Tualle-Brouri, and Ph. Grangier, Phys. Rev. Lett. 93, 130409 (2004).

[6] C. Cohen-Tannoudji, B. Diu, and F. Laloë, Quantum Mechanics (John Wiley and Sons, NY, and Hermann, Paris,
1977).

[7] A.M. Gleason, J. Math. Mech. 6, 885 (1957).

[8] S. Kochen and E.P. Specker, J. Math. Mech. 17, 59 (1967).

[9] W.K. Wootters and W.H. Zurek, Nature 299, 802 (1982); D. Dieks, Phys. Lett. A 92, 271 (1982); H.P. Yuen, Phys. Lett. A 113, 405 (1986).

[10] D.M. Greenberger, M.A. Horne, and A. Zeilinger, in Bell's Theorem, Quantum Theory, and Conceptions of the Universe, edited by M. Kafatos (Kluwer Academic, Dordrecht, The Netherlands, 1989).

[11] M.H. Partovi, Phys. Lett. A 137, 440 (1989).

[12] L. Hardy, Phys. Rev. Lett. 71, 1665 (1993).

[13] R. Olkiewicz, Comm. Math. Phys. 208, 245 (1999).

[14] A.K. Pati and S.L. Braunstein, Nature 404, 131 (2000); ibid., quant-ph/0007121

[15] R. Olkiewicz, Ann. Phys. 286, 10 (2000).

[16] L. Hardy, quant-ph/0101012

[17] K. Dietz, J. Phys. A - Math. Gen. 35, 10573 (2002); ibid., 36, 5595 (2003); ibid., 36, L45 (2003).

[18] C.M. Caves, C.A. Fuchs, K. Manne, and J.M. Renes, Found. Phys. 34, 193 (2004), and references therein. 
[19] A. Peres, Quantum Theory: Concepts and Methods (Kluwer, Dordrecht, 1995), p. 278.

[20] An antiunitary operator can be always written in the form $U \mathcal{C}$, where $U$ is a unitary operator and $\mathcal{C}$ is complex conjugation in the choosen basis.

[21] K. Huang, Statistical Mechanics, (John Wiley \& Sons, New York, 1987).

[22] D. Welsh, Codes and Cryptography, (Clarendon Press, Oxford, 1989).

[23] A. Wehrl, Rev. Mod. Phys. 50, 221 (1978).

[24] J. von Neumann, Gött. Nachr., 271 (1927).

[25] E.P. Wigner, Group Theory, (Academic Press, New York, 1959).

[26] G. Emch and C. Piron, J. Math. Phys. 4, 469 (1963).

[27] N. Gisin, Am. J. Phys. 61, 86 (1993).

[28] There are many ways in which one can quantify entanglement. A partial list includes 29].

[29] C.H. Bennett, D.P. DiVincenzo, J.A. Smolin, and W.K. Wootters, Phys. Rev. A 54, 3824 (1996); V. Vedral, M.B. Plenio, M.A. Rippin, and P.L. Knight, Phys. Rev. Lett 78, 2275 (1997); D.P. DiVincenzo, C.A. Fuchs, H. Mabuchi, J.A. Smolin, A. Thapliyal, A. Uhlmann, quant-ph/9803033 T. Laustsen, F. Verstraete, and S.J. van Enk, QIC 3, 64 (2003); M.A. Nielsen, Phys. Rev. Lett. 83, 436 (1999); G. Vidal, J. Mod. Opt. 47, 355
(2000); D. Jonathan and M.B. Plenio, Phys. Rev. Lett. 831455 (1999); M. Horodecki, A. Sen(De), and U. Sen, Phys. Rev. A 70, 052326 (2004); M. Horodecki, QIC 1, 7 (2001).

[30] C.H. Bennett, H.J. Bernstein, S. Popescu, and B. Schumacher, Phys. Rev. A 53, 2046 (1996); S. Popescu and D. Rohrlich, Phys. Rev. A 56, 3219 (1997); G. Vidal, J. Mod. Opt. 47, 355 (2000); M. Horodecki, P. Horodecki, R. Horodecki, Phys. Rev. Lett. 84, 2014 (2000).

[31] E. Schmidt, Math. Ann. 63, 433ff (1907).

[32] See e.g. Ref. [21], p. 73.

[33] Note that the usual statement of the Boltzmann $H$ theorem is with the function $H(t)$, without the minus sign.

[34] See e.g. Ref. 21], p. 62.

[35] E.A. Calzetta and B.L. Hu, Phys. Rev. D 37, 2878 (1988); ibid. 68065027 (2003), and references therein.

[36] See e.g. Ref. 21], p. 64.

[37] Ref. [19], p. 267.

[38] S. Popescu and D. Rohrlich, Phys. Rev. A 56, R3319 (1997); M. B. Plenio and V. Vedral, Cont. Phys. 39, 431 (1998); M. Horodecki and R. Horodecki, Phys. Lett. A 244, 473 (1998); P. Horodecki, M. Horodecki and R. Horodecki, Acta Phys. Slovaca 48, 141 (1998). 